\title{
La France " progressiste » contre la Russie « despotique » : l'image de la Russie dans les pages du Moniteur universel en 1789-1793
}

\author{
Natalia Vochtchinskaya \\ chercheure indépendante \\ nvochtchinskaya@yahoo.fr
}

\begin{abstract}
This article considers some ideological, political, religious and cultural aspects of the image of Russia in the French newspaper Moniteur universel, mostly in the period between 1789 and 1793. A comparative analysis of the information contained in the 'Politics' section of the newspaper gives an idea of the attitudes and opinions of the editors and contributors vis-à-vis Russia and other countries, which were shaped by the pressure of the diverse and rapidly changing circumstances in French political life during the period in question. The author also proposes a classification scale of news related to Russia ranged in accordance with their content, subjects, importance and regularity. The table in the Annex contains a calculation of the references to different states for the years 1790, 1793 and 1796 produced by the author in order to correct the data presented in nineteenth-century Indices.
\end{abstract}

Mots clé

presse française; Révolution française; Le Moniteur universel; image de la Russie ; despotisme ; Catherine II ; Paul I ${ }^{\mathrm{er}}$

Le but de cette publication est d'étudier les nouvelles de La Gazette nationale, ou le Moniteur Universel (infra - MU) concernant la Russie et de les comparer avec celles relatives aux autres pays. Nous proposons une analyse des «sujets russes » de ce périodique.

Nous n'y avons répertorié que les mentions au titre «Russie» dans la rubrique "Politique » qui occupe tout ou une partie de la première page et dans laquelle on trouve des nouvelles provenant de différents pays. Les nouvelles liées à la Russie apparaissent également dans des articles concernant d'autres pays, ce qui augmente l'importance de ce sujet, mais ces mentions n'ont pas été prises en compte dans les calculs. Les Index de ce périodique, rédigés en 1802 et $1870^{1}$, ne donnent pas une idée précise de son contenu, bien que celui de 1870 ait été largement augmenté en comparaison avec le premier (19 575 articles contre 12 908). Aucun d'eux n'indique par exemple les noms des grands-ducs Alexandre et Constantin, petit-fils de Catherine II, bien qu'ils apparaissent dans les nouvelles ${ }^{2}$. De surcroît, les mentions de la «Russie» proprement dite ne sont pas toutes

\footnotetext{
${ }^{1}$ Révolution française. Table alphabétique du Moniteur, depuis 1787 jusqu'à l'an 8 de la République (1799), Paris, Girardin, 1802 ; Réimpression de l'ancien Moniteur (mai 1789-novembre 1799). Table rédigée et collationnée par M. A. Ray, Paris, Henri Plon, 1870.

${ }^{2}$ Voir, par exemple, la nouvelle sur le futur mariage d'Alexandre (le 6 mars 1793, n ${ }^{\circ} 65$ ). Selon l'Index le plus complet de 1870 , pendant la période de mai 1789 à novembre 1799 , une seule mention de Moscou apparaît dans les pages de l'édition, autant qu'Arkhangelsk, ce qui ne reflète pas du tout la réalité.
} 
incluses dans ces Index : les auteurs ont souvent omis les cas où elle était citée dans les nouvelles des autres pays quand bien même elle était le sujet central de la publication. Par ailleurs, les références se croisent souvent, en créant des doublons de différents articles. A titre d'exemple, l'auteur cite deux fois la même nouvelle dans les articles intitulés « Russie » et «Artois » (l'arrivée du comte d'Artois en Russie), mais on ne trouve la mention de la reconnaissance de son titre de lieutenant-général par la cour russe que dans l'article "Artois», et non dans l'article "Catherine II $»^{3}$. Ainsi, il est presque impossible de comprendre le taux de présence des sujets russes dans les pages du $M U$ en s'appuyant uniquement sur les Index, c'est pourquoi nous avons fait notre propre calcul au sein de la rubrique « Politique » pour les années 1790, 1793 et 1796 (voir l'Annexe).

En analysant les nouvelles « russes » dans le $M U$ en 1789-1793, nous avons constaté que la Russie, malgré son rôle important dans les affaires européennes, n’apparaît qu'assez rarement dans les pages du journal en comparaison avec certains autres pays (voir l'annexe ci-dessous). Par exemple, « Russie » apparaît dans le titre de l'article un nombre insignifiant de fois par rapport à l'Allemagne, ou à l'Angleterre, en tête de liste, quelle que soit la méthode utilisée. En effet, si on prend en considération les mentions de "Russie » dans les rubriques consacrées aux autres pays, surtout «Turquie ", "Pologne », "Suède ", ce nombre sera bien plus élevé ; cependant, en appliquant la même méthode de calcul pour les autres pays, leur fréquence augmente également, et celle de la Russie reste toujours inférieure.

A notre avis, cet état de fait s'explique par les causes suivantes (à commencer par les moins importantes) :

1. La distance séparant la France et la Russie peut expliquer en partie le nombre considérable des nouvelles russes arrivant indirectement, via d'autres pays européens plus proches de la Russie. Cependant, cet argument s'avère facile à réfuter : en juilletdécembre 1796, la rubrique les « Etats-Unis d'Amérique » apparaît 7 fois, le " Danemark » - 6, l'« Espagne »- 15, la « Pologne »- 5, la « Russie» $4^{4}$.

2. L'hostilité entre la France et la Russie et la rupture des relations diplomatiques, économiques et politiques ont abouti à une censure éditoriale. Là encore, cette hypothèse se heurte à certains exemples : le fait qu'en 1793 la « Russie » figure 27 fois sur les pages du $M U$, contre 76 pour l'« Angleterre », ennemi juré de la France ${ }^{5}$.

3. Les régimes politiques russe et français divergent de plus en plus, et ce fossé influence la quantité et la qualité des nouvelles russes. Cependant, les nouvelles d'autres puissances monarchistes et anti-françaises sont publiées plus souvent que celles provenant de Russie.

\footnotetext{
${ }^{3}$ Réimpression de l'ancien Moniteur (mai 1789-novembre 1799). Table rédigée et collationnée par M.A. Ray, Paris, Henri Plon, 1870 , t. 30, p. 42, 195 ; t. 31, p. 400.

${ }^{4}$ Il faut tenir compte du fait que l'année 1796 n'est pas très représentative ; il semble que le journal, surtout pendant la deuxième moitié de l'année, ne s'intéresse que très peu au reste du monde, préférant les nouvelles de l'armée d'Italie, dont on lit de longues narrations des succès du général Bonaparte avec des commentaires extatiques. En juillet-décembre, la rubrique l'« Italie » remporte la palme avec 75 citations (une croissance de deux fois et demie en comparaison avec la même période de 1793), elle est la plus prolixe de toutes. La rédaction du journal est si préoccupée par les affaires italiennes et la nécessité de porter la guerre contre l'Angleterre, qu'elle remarque à peine la mort de Catherine II le 6 novembre 1796. On ne trouve aucun article dédié à ce sujet dans les mois suivants, seulement quelques phrases vagues insérées dans les autres nouvelles, qui permettent de deviner avec beaucoup de difficulté la disparition de l'impératrice.

${ }^{5}$ Voir point $\mathrm{n}^{\circ} 5$.
} 
4. La Russie, malgré sa participation assez active dans l'alliance anti-française, reste pour l'opinion publique en France au second plan des menaces politiques et militaires, cédant la première place aux autres pays, avec l'Angleterre presque toujours en tête.

5. Les nouvelles russes étaient parfois trop étroitement liées à celles des autres pays, ainsi, les éditeurs du $M U$ se bornent à l'information concernant ces derniers, dans laquelle la Russie occupe une place importante (cas de la Suède en 1790 et de la Pologne en 1793, par exemple).

6. La Russie est une puissance assez renfermée et n'offrant que peu d'informations sur sa vie intérieure, que ce soit par les canaux officiels, ou par sa presse. Les décisions et les intentions du gouvernement russe, l'état de ses finances et ses forces militaires (les sujets les plus "passionnants» pour l'Europe) sont dissimulés, la presse russe est censurée, et l'Europe se nourrit de rumeurs provenant de sources souvent indirectes. Ce n'est pas le cas pour l'Allemagne dont la presse est développée et surtout pour l'Angleterre, où existent des débats parlementaires immédiatement publiés et commentés dans de nombreux journaux et «feuilles ». Le $M U$ profite beaucoup de la "démocratie » anglaise, et puise dans cette information pour tirer des conclusions et livrer des commentaires variés, y compris sur des questions militaires et financières. La « discrétion » du système russe ne favorise pas la diffusion d'informations vérifiées, le $M U$ doit donc se contenter de nouvelles incomplètes empruntées aux journaux nationaux.

Si l'on en juge par le contenu des nouvelles «russes", l'image de l'Empire de Catherine II est celle d'un pays où il ne se passe rien d'intéressant. Les informations sont soit insignifiantes, soit très officielles, soit purement mondaines.

On peut les classer grâce aux catégories suivantes :

1. Les nouvelles « climatiques » (par exemple, l'état de la Néva en hiver et en été, les dates de la prise des glaces et de la débâcle : une rengaine qui devient inopportune en l'absence presque complète de nouvelles vraiment importantes. Cependant, il faut prendre en compte que ces informations étaient utiles pour la navigation et le commerce, avant la rupture des relations diplomatiques et commerciales entre les deux pays en février 1793).

2. Les nouvelles de la cour : distribution des décorations, promotions et autres " grâces » impériales, soupers, bals, fêtes, baptêmes et autres cérémonies, déplacements de l'impératrice (ses allers-retours entre Saint-Pétersbourg et ses autres résidences), etc.

3. Les nouvelles « militaires » et « maritimes » : position et mouvements des troupes russes, leur nombre, leurs victoires et leurs défaites, lancement des navires et autres opérations navales ; pertes humaines et matérielles de toutes sortes (prisonniers, morts, navires, etc.) ; affectations des officiers, nominations, prétentions militaires du gouvernement russe, etc.

4. Les nouvelles diplomatiques : les nominations et les affectations des ambassadeurs, russes et étrangers, les « circulaires » données aux ambassadeurs russes par l'impératrice et distribuées dans les légations russes, les intentions diplomatiques de la Cour russe, les textes des traités et ordonnances impériales relatives aux affaires étrangères, etc.

5. Les nouvelles concernant la politique internationale de la Russie, peu nombreuses si on déduit les points précédents : la politique russe dans les différents pays au moment des crises (la Suède, la Pologne, la Turquie) ; la position russe à l'égard de la France révolutionnaire ; les alliances conclues et rompues ; les relations de la cour russe avec les gouvernements européens; les alliances matrimoniales "politiques » avec d'autres cours (cas du mariage projeté, mais non abouti, en 1796, du futur Gustave IV de Suède et d'Alexandra, petite-fille de Catherine II) ; etc. 
6. Les nouvelles de la vie intérieure de la Russie, qui sont elles aussi très liées aux sujets susmentionnés. Ceux-ci mis à part, restent les questions où la France est directement concernée : les mesures du gouvernement russe prises contre la diffusion des idées révolutionnaires à l'intérieur de l'Empire; sa politique à l'égard des Français en Russie, etc. Parfois on voit des nouvelles locales importantes pour des questions militaires et politiques (incendie du port d'Arkhangelsk, troubles à Moscou à l'annonce de la conscription, etc.).

7. Les publications des actes officiels du gouvernement russe importants pour l'Europe (textes des traités, ordonnances, oukases, etc.).

L'approche des affaires russes dans les publications du $M U$ n'est pas toujours la même au cours de la période étudiée. Elle ne dépend pas complètement de la conjoncture politique, mais aussi de l'évolution même de la rédaction. Fondé en 1789, le $M U$ devient très progressivement un «journal de propagande " sur les sujets internationaux ${ }^{6}$, se laissant emporter par le mouvement révolutionnaire. L'exactitude, la rigueur, l'absence d'émotion et de commentaire caractérisent ce périodique à ses débuts. Ainsi, l'année 1790 se distingue vraiment de 1793 ou 1796 marquées par une verve hostile et propagandiste. On a l'impression que les rédacteurs lancent des ballons d'essai comme, par exemple, les « lettres des particuliers ». Ce moyen sera très utilisé plus tard quand le journal publiera les lettres des patriotes polonais en 1793 en soulignant leur bravoure face à la férocité et à l'injustice des usurpateurs ${ }^{7}$. Cette méthode consiste à personnaliser l'information sous forme de «témoignages » visant à inspirer confiance aux lecteurs, tout en présentant les faits sous un angle favorable à la France.

En ce qui concerne la Russie, le $M U$ n'emploie pas cet instrument de persuasion : on n'y trouve pas de lettres « russes ${ }^{8}$. En 1790, l'information sur l'Empire est présentée avec impartialité, ainsi que celle des autres pays. Cela s'explique sans doute aussi par le régime toujours monarchiste de la France au début de la Révolution. Ce n'est que plus tard, quand la France s'écarte du reste de l'Europe, que le régime monarchique devient un objet de blâme quelles que soient les nouvelles. Les publications de l'année 1790 sur la France constituent un mélange bizarre de nouveautés et de vestiges de l'Ancien Régime ; certaines nouvelles sont très similaires à celles des autres monarchies européennes, y

\footnotetext{
${ }^{6}$ Ce n'était pas toujours le cas pour l'interprétation des affaires intérieures : ici, la rédaction prend vite un ton de propagande et décrit avec enthousiasme et sous un jour favorable toutes les "nouveautés » introduites par la Révolution. Elle soulignait le progrès des idées et des institutions nouvelles, le soutien par le peuple et les forces « progressistes » de la nouvelle situation politique en France. L'information régulière sur le "patriotisme » et le "sacrifice " des citoyens était destinée à enflammer les esprits, à justifier les changements, à prouver leur nécessité et à recruter des partisans du nouvel ordre. Cette ligne éditoriale mène à l'apparition de la rubrique intitulée sans équivoque « Patriotisme » et à une propagande de plus en plus « cousue de fil blanc».

${ }^{7}$ Beaucoup de ces lettres sont rédigées au sein de la rédaction du périodique, très loin des contrées polonaises.

${ }^{8}$ Les journaux russes publient des « lettres de la France». Les correspondants y décrivent les troubles en France, leurs commentaires sont opposés à ceux du $M U$ : les «actes de patriotisme » sont interprétés comme des émeutes sanglantes, l'émergence de la conscience populaire comme la ruine de l'ordre sociale par des brigands; les grands acteurs de la Révolution sont accablés d'injures comme dilettantes, comédiens, ivrognes ou tout simplement fous. Même les historiens russes tiennent dès le XIX $\mathrm{X}^{\mathrm{e}}$ siècle ces opinions pour extrêmement partiales et injustes, en remarquant «l'étroitesse d'esprit » des informateurs français, dont «l'argumentation contre le libéralisme et les doctrines de l'Assemblée Nationale se borne à l'ironie prétentieuse et aux âcres jurons ", А. Брикнер, “'Санкт-Петербургские ведомости' во время Французской революции », Древняя и новая Россия [A. Brikner, «Sankt-Péterbourgskié Védomosti pendant la Révolution Française », La Russie ancienne et moderne], 1876, t. I, p. 83, 84.
} 
compris la Russie 9 . On ne trouve pas beaucoup de différence entre les nouvelles des célébrations : les anniversaires des rois du Danemark ou de Suède sont pareils aux fêtes à la cour russe, le "modèle » étant partout similaire : un "grand gala », un bal, une table avec de nombreux couverts... De même, la rubrique "France » commence toujours par des nouvelles de la vie de la famille royale ${ }^{10}$. Rien ne distingue ces rubriques de celles des autres familles régnantes : communions des enfants de Louis XVI, présence de sa famille aux messes, visites et hommages rendus à Sa Majesté, etc. Parmi ces textes, on trouve une information curieuse et non politique liée à la Russie, l'exemple est presque exceptionnel : «Le 18, M. de Lesseps, Consul de France, a eu l'honneur de présenter au Roi, à la Reine \& à la Famille Royale, le Journal historique de son Voyage de la Baie d'Avatcha, au Kamtzcatka, en France... » (le 21 avril 1790, nº11 $)^{11}$.

\section{La Russie et d'autres pays : la «barbarie» monarchique et la "modernisation» révolutionnaire}

Ce qui distingue la Russie d'autres pays monarchiques, ce sont les informations relatives aux grâces accordées par Catherine II, habituelles pour la Russie de l'époque, mais étonnantes pour un regard étranger : ce sont des cadeaux « vivants ». Par exemple, en communiquant la satisfaction de l'impératrice à l'égard des actions de la flotte de l'amiral V. Tchitchagov contre les Suédois, le journal informe les lecteurs français le 18 juin 1790 que «S.M. lui a fait présent de 14 ooo Paysans, \& l'a décoré de l'Ordre de S. André ». Les fils de l'amiral obtiennent des ordres, des promotions, des épées d'or et des tabatières d'une valeur de 1500 roubles $\left(n^{\circ} 169\right)$. L'énumération des gens parmi les objets est alors courante en Russie et typique des périodiques russes, où les propriétaires proposent sans gêne d'acheter des carrosses, des livres, des maisons, des chevaux, des chiens et des serfs. De telles pratiques contraires aux idéaux d'égalité et de fraternité devaient être frappantes pour l'opinion française et faire apparaître la Russie sous un jour

\footnotetext{
9 A l'exception peut-être de l'Angleterre: le journal français informe ses lecteurs sur les nouvelles «mondaines» (les prix des huitres à Londres, par exemple), et également sur des événements atypiques pour des monarchies non-constitutionnelles. Grâce à la «liberté anglaise », la vie politique, sociale et journalistique de ce pays est bien mieux présentée que pour les monarchies absolutistes en « hibernation »; grâce à la presse anglaise, les nouvelles arrivant en France d'Angleterre sont aussi plus variées que celles d'autres pays.

${ }^{10}$ Les nouvelles «royales» sont suivies des décrets de l'Assemblée Nationale et du roi, ainsi que des nouvelles régionales qui contrastent avec celles de la vie de Louis XVI, évoquant les « assemblées partielles de la Commune », la « Milice Nationale de Grenoble », etc.

"Les nouvelles "géographiques" sur la Russie, quoique rares, sont les plus répandues après les «actualités » politiques, contrairement aux nouvelles culturelles, littéraires, etc., presque inexistantes. Il nous est difficile d'accepter l'assertion selon laquelle « une grande partie de tous les articles et entrefilets sur la Russie [dans le $M U$ ] contenaient justement des renseignements sur sa vie en temps de paix, son climat, les sciences, la société russe, les éminents hommes d'Etat et certains célèbres étrangers au service russe.» А.А. Митрофанов, Ж. Оливье-Шахновская, «Образ в России в прессе революционной Франции 1789-1792 гг. (на примере газеты «Монитёр»)», Европа: Международный альманах [A. Mitrofanov, J. Ollivier-Chakhnovskaïa, «L'image de la Russie dans la presse révolutionnaire de France en 1789-1792 (le cas du « Moniteur Universel ») », Europe: Almanach international], Tioumen, 2005, ${ }^{\circ} 5$, p. 88. La diversité des aspects de la vie sociale en Russie n'est que très peu reflétée dans le $M U$. Les journaux russes, construits sur le modèle de ceux de l'Europe, se focalisent aussi sur la vie politique : les nouvelles de l'étranger sur la vie sociale, le climat, les découvertes géographiques, etc. y apparaissent rarement. La France n'y occupe pas une grande place jusqu'au début de la Révolution, cédant le pas aux pays voisins, surtout à la Suède et à la Turquie. А. Кирпичников, «'Московские ведомости' и начало Французской революции, Исторический вестник», Исторический вестник [A. Kirpitchnikov, "'Moskovskie Vedomosti' » et le début de la Révolution Française », Istoritcheski vestnik], 1882, t. IX, p. 462.
} 
encore plus déprécié qu'autrefois ${ }^{12}$. Le prix de la seule tabatière mentionnée correspondait au revenu annuel d'un domaine de 300 paysans; avec le «cadeau » de 14 ooo serfs, le revenu de l'amiral augmente de 70 ooo roubles (seuls 700 à 800 nobles en Russie possèdent alors plus de 1500 paysans. Un seul habit de "gala » du favori et ministre Grigorii Potemkine coûtait 200 ooo roubles ${ }^{13}$, c'est-à-dire, trois revenus annuels de Tchitchagov avec ces 14 ooo paysans).

Evidemment, le « retard » politique et la civilisation archaïque de la Russie n'est pas aussi évident que celui de la Porte Ottomane, d'où arrive cette nouvelle le 8 février 1790 : «Un Courrier est arrivé, le 12, à Constantinople, portant la tête du Gouverneur d'Akiermenn, décapité par les ordres du nouveau Grand-Visir, quoiqu'il fût bien prouvé qu'il n'avoit pas pu se défendre, puisqu'il n'avoit avec lui que cent hommes, \& qu'il manquoit d'argent, de vivres \& de munitions $»^{14}\left(\mathrm{n}^{\circ} 39\right)$. Il est important de noter que les «barbaries » russes et turces ne jouissent d'aucun commentaire ni d'aucune remarque de la part du $M U$ : au début, répétons-le, la présentation des nouvelles est très neutre.

Le $M U$ publie plus volontiers les nouvelles « progressistes » qui s'accordent mieux avec le régime républicain et "l'air de liberté » qui règne en France. C'est pourquoi les informations provenant des Etats-Unis d'Amérique sont longues et imprégnées de la même verve libérale que celles de France, avec " adresses au Public », discussions libres, «sociétés » des citoyens, etc. Les sténographies des séances de la diète polonaise sont également assez fréquentes, et, à notre avis, non seulement en raison de l'importance accordée à ce pays dans la politique française, mais parce que cette information est plus "vivante » que les nouvelles stéréotypées sur les déplacements de souverains ou la débâcle des fleuves. L'agitation de la vie politique, les discussions et les batailles verbales sont plus intéressantes pour la France de l'époque que l'immobilisme monarchiste de pays plus ou moins fermés.

Avec les mutations intérieures et les événements extérieurs, la rédaction évolue. Cela devient surtout évident en 1792-1793, avec la formation de la Première coalition, l'avènement de la dictature jacobine et le partage de la Pologne. La France se sent de plus en plus isolée, entourée d'ennemis, et le $M U$ commence à accomplir le rôle de soutien de la Révolution, en justifiant les démarches françaises et en accusant l'étranger de manière agressive et parfois maladroite.

L'Angleterre ne présente alors d'intérêt pour le $M U$ que du point de vue de ses forces maritimes et de ses intentions à l'égard de la France, à l'instar des autres pays de la coalition antifrançaise. Parallèlement, le journal s'intéresse à toute nouvelle étrangère

\footnotetext{
${ }^{12}$ Avant la Révolution, les Français considéraient le servage russe comme un héritage du féodalisme et même de l'esclavage romain. En analysant ces annonces un siècle plus tard, un historien russe répétera les propos des voyageurs français du XVIII ${ }^{\mathrm{e}}$ siècle choqués par cette pratique, en parlant du «commerce inhumain des gens parmi le bétail et les objets inanimés », ibid., p. 456.

${ }^{13}$ Н.Я. Эйдельман, Грань веков [Natan Eidelman, A la frontière des deux siècles], Moscou, 2004, p. 29-30. Si en 1815 les ricaneurs français publient une caricature de Napoléon avec l'inscription : « Passant, ah ne plains pas mon sort Si je vivais, tu serais mort » («Projet de Tombeau », BNF, Collection Hennin, Tome 157, maiaoût 1815, G165 UU 7, 13 767), des railleurs russes « honorent » le prince Potemkine de cette épitaphe plaisante: «Passant, remercie le Créateur Très-Haut que la Russie sous lui n'a pas été ruinée » (« Прохожий, помоли Всевышнего Творца, что сей не разорил Россию до конца »).

${ }^{14}$ Il s'agit des conséquences de la prise de la forteresse turque Akkerman par les troupes russes le 9 octobre 1789. Le gouverneur de cette forteresse, Taifour Pacha, n'est pas vraiment coupable de la reddition : afin d'éviter la bataille contre les navires russes, le 23 septembre, la flotte turque part de Gadjibée pour Constantinople, en laissant Akkerman sans couverture. Le sultan Sélim III s'oppose aux négociations avec les Russes et remplace son Grand Vizir pacifique par Cezayirli Gâzi Hasan Pacha mettant un terme aux négociations secrètes entre son Grand Vizir, le ministre Potemkine et le feld-maréchal autrichien E.G. Laudon.
} 
avantageuse à l'égard de la France : les conflits politiques en Suède, l'exercice libre de la profession des poissonniers et des bouchers de Bruxelles, les adresses de la "société des amis de la Liberté et de l'Egalité » des Pays-Bas, les lettres de soutien à la Révolution française prétendument venues de toutes les contrées, y compris monarchistes (de la Suède, par exemple), etc.

L'éclectisme politique en France, de règle au début de la Révolution, disparaît, le contenu du $M U$ en est la preuve: les sténogrammes prolixes des débats dans la Convention nationale sur le sort de Louis Capet, puis les débats sur Marie-Antoinette, sont orientés, les descriptions détaillées de leurs dernières heures en témoignent. En présentant aux lecteurs le testament du roi décapité, le périodique écrit que « Louis ne fut pas un instant de bonne foi, que tous les serments ont été des parjures ; que la révolution ne fut jamais autre chose pour lui que la cause nécessaire d'une contre-révolution » (le 28 janvier $1793, \mathrm{n}^{\circ} 28$ ). Les commentaires soulignent la « mauvaise foi » du roi et ne relèvent pas la dignité et la générosité qui percent à travers les lignes de son testament. A propos de ces mêmes événements, le journal n'oublie pas d'insérer une description du deuil pris en Angleterre à la nouvelle de la mort de Louis XVI (le 2 février $1793, \mathrm{n}^{\circ} 33$ ). Mais le 4 février on émet des doutes à ce propos : «Londres est, au moins en apparence, dans une consternation générale... » $\left(\mathrm{n}^{\circ} 35\right)$. Selon le périodique, Copenhague reste assez indifférent à l'«événement du 21 janvier», une description vague et un peu «pudique » de l'exécution du roi (le 3 mars 1793, $\mathrm{n}^{\circ}$ 62). «Des cris de fureur contre la France et les Français » à Madrid, "à la nouvelle de supplice de Louis XVI », sont expliqués par le nombre important des « émigrés, des moines, des aristocrates » (le 4 mars 1793, n63). La réaction de la cour russe est présentée sèchement et sans émotion particulière malgré la formulation brutale de l'oukase sur le deuil, qui évoque des « factieux » qui ont « commis un assassinat impie ». Le $M U$ mentionne en passant : " ce sont les expressions dont on s'est servi », et met un point final à l'article (le 26 mars 1793, n85).

\section{Les affaires polonaises}

En 1793, la Russie apparaît surtout à propos des nouvelles venues de Pologne et de Turquie, mis à part les informations récurrentes sur ses armées et sur sa flotte. Souvent les nouvelles polonaises sont présentées comme provenant de Pologne même et sont ainsi loin d'être favorables à la Russie. Les propos deviennent de plus en plus brusques et accablants pour Catherine II : on lui reproche en vrac son despotisme, son intervention dans les affaires d'un autre pays, l'annulation de la Constitution de 1791, la nomination de ses créatures, la tentative de « fixer le joug de la Russie sur la tête des Polonais » (le 30 janvier 1793, $\mathrm{n}^{\circ} 30$ ). On souligne sa perfidie : «Il faut être l'impératrice de Russie pour combiner tant d'infortunes sur un seul peuple, et l'appeler encore son allié et son ami » (le 19 février $1793, \mathrm{n}^{\circ}{ }^{\circ}$ ) ; on rappelle tous ses anciens péchés : "L'impératrice de Russie, cette femme qui a attaché son nom à tous les grands attentats qui se sont commis en Europe, depuis qu'un meurtre l'a couronnée ${ }^{15}$, ne permettra point aux Polonais d'échapper au sort qui leur est préparé » (le 6 mars 1793, n65). L'épithète la plus innocente et la plus fréquente que l'on accole au nom de Catherine est « ambitieuse ». Le $M U$ préfère oublier que son propre roi vient d'être guillotiné ; mais cette exécution est votée « légitimement » et n'est donc pas comparable avec un coup d'Etat ou un assassinat « clandestin ». La ligne partisane du journal tend à dénigrer les ennemis par tous les

\footnotetext{
${ }^{15}$ Il s'agit du coup d'Etat de 1762 et de la mort violente de l'empereur Pierre III, époux de Catherine. Ce sujet est évoqué par le $M U$ à plusieurs reprises, afin de montrer l'immoralité absolue de l'impératrice.
} 
moyens possibles, quitte à accentuer leur mauvaise foi et leurs crimes, tout en justifiant la position française. Si l'on croit Charles Masson, Catherine II était peu sensible aux attaques personnelles du $M U$ :

Il est à remarquer que, sous le règne de Catherine, la Russie fut, pendant un tems, le seul pays de l'Europe où les papiers français ne fussent pas défendus. Le Moniteur ayant parlé plusieurs fois de l'impératrice, et surtout de Paul et de sa cour, Catherine donna ordre qu'on ne distribuât plus le Moniteur qu'après qu'elle l'auroit parcouru. Quelques semaines après, elle y trouva un article où elle étoit qualifiée de Messaline du Nord, etc. L'ayant lu, elle dit : Puisque cela ne regarde que moi, qu'on le distribue ${ }^{16}$.

Au même moment, le roi Stanislas perdant le pouvoir devant le parti « patriote » est lui aussi dénigré et insulté, ce qui n’a pas été le cas dans les années précédentes. On parle de sa faiblesse face à des "oppresseurs » : il est devenu un "moins que rien, puisqu'il est joué, méprisé, dédaigné » (le 17 janvier 1793, $\mathrm{n}^{\circ}{ }_{17}$ ), il "est allé cacher sa honte à la campagne » (le 16 février 1793, n 47 ), il « emploie le loisir que les malheurs de sa patrie lui laissent, en bals, en spectacles, et mérite maintenant tout l'opprobre qui a succédé pour lui à tant de vénération qu'il ne méritait pas » (le 6 mars 1793, n65). La Confédération est appelée «régime absurde et despotique» (le 30 janvier 1793, $\mathrm{n}^{\circ} 30$ ) ; Stanislas Félix Potocki est qualifié d' « agent fidèle » de la Russie (le 2 février, $\mathrm{n}^{\circ} 33$ ), dont les services sont bien rémunérés. La Pologne et les Polonais méritent souvent l'adjectif « malheureux », les nouvelles polonaises sont données sur un registre sentimental, on parle de leurs "âmes déchirées » et des larmes versées par les Polonais, avec des exclamations comme : «Et tout ceci se passe à la fin du dix-huitième siècle ! » (Le 10 mai 1793, $\mathrm{n}^{\circ}{ }_{130}$ ). La rédaction ne rate pas une occasion pour dénigrer la Russie et le parti russe en Pologne, dans un style plein d'ironie. Ainsi, "souveraine" (à propos de Catherine II, souvent précédé du possessif «sa» quand on parle des Polonais attachés aux usurpateurs russes), «protectrice» (à propos de la Confédération soutenue par la Russie, dont les actions mènent à l'intervention des troupes russes à Varsovie en 1792 et à l'annulation de la Constitution polonaise de 1791) sont mis en italique ${ }^{17}$. Il s'agit bien d'un "sarcasme typographique », les exemples suivants le prouvent : "Ainsi les ordres ont été donnés pour que ce dernier [le comte d'Artois] fût traité conformément à ce qu'on appelle encore ici [à Pétersbourg] son rang et sa naissance (le 13 mai 1793, n¹31) ; "M. d'Artois est de retour de son pèlerinage auprès de la Sémiramis du Nord » (le 29 mai 1793, n 149$)^{18}$.

\footnotetext{
${ }^{16}$ Masson Ch.F.Ph., Mémoires secrets sur la Russie, Paris, 180o-1804, t. II, p. 186-187. Plus loin, Masson tend à exagérer la «tolérance » de l'impératrice en comparaison avec d'autres monarques de l'Europe. Cependant, les réactions et les mesures prises par Catherine contre la « contagion française » dans son Empire n'ont pas toujours été aussi clémentes que veut le faire croire le passage cité.

${ }^{17}$ La Russie ou la Pologne ne sont pas des exceptions : tous les ennemis de la France sont traités de cette manière. Ainsi Calonne, qualifié en italique de «prédicateur de croisades" (le 2 février 1793, $\mathrm{n}^{\circ} 33$ ) et l'expulsion des Français par la noblesse hongroise définie de manière caustique comme «un acte de patriotisme » (le 4 mars $1793, \mathrm{n}^{\circ} 63$ ).

${ }^{18}$ Cette information est placée dans la rubrique "Danemark», ce qui conforte l'hypothèse que les rédacteurs du $M U$ modifient la présentation des nouvelles arrivant de tous les pays, en y ajoutant leur propre touche : il est difficile d'imaginer qu'une source danoise ait pu ironiquement appeler le séjour d'Artois en Russie « pèlerinage » et Catherine II, « la Sémiramis du Nord ». La liberté de la presse introduite au Danemark par l'ordonnance du 4 octobre 1770 suscite une réaction enthousiaste de Voltaire et la Révolution Française à ses débuts ajoute des sympathisants au Danemark. Mais, dès la fin de 1790, le gouvernement danois impose la censure, et celle-ci est encore aggravée après l'exécution de Louis XVI qui génère des mesures sévères et l'exil des publicistes défendant la Révolution. La plupart d'eux trouvent asile en France (P.A. Heiberg, M.C. Bruun); la liberté de la presse, jamais abrogée, est cependant considérablement réduite en 1799. Johny Leisner, La Constitution du Danemark. 150 ans. La France et la
} 


\section{La situation des émigrés français en Russie}

Un autre sujet important ressort dans les pages du $M U$ en cette année 1793 : le sort des émigrés et leur accueil dans les pays d'asile. La Russie est citée très fréquemment, au sujet du serment infligé aux résidants français dans l'Empire, selon l'oukase du 8 février. Ce thème a déjà été couvert plus tôt et toujours avec ironie ( "Catherine s'empresse d'accueillir les débris de l'aristocratie française » (le 2 février, $\left.\mathrm{n}^{\circ} 33\right)$ ). La messe pour Louis XVI commandée par la cour russe dans une église catholique de Saint-Pétersbourg qui a coûté, selon les rumeurs, plus de cinq mille roubles, mérite un commentaire caustique du $M U$ : «Il vaudrait sans doute mieux de payer ses dettes et de venir au secours de ceux qu'on a ruinés... » (le $1^{\mathrm{er}}$ mai $\left.1793, \mathrm{n}^{\circ}{ }^{121}\right)$. Mais parfois on présente ce même sujet sobrement, en citant les nouvelles nominations des émigrés à la cour russe, l'aide financière à ceux employés au service de la Russie ${ }^{19}$, et même le contenu des oukases restreignant l'entrée des Français en Russie (le 26 mars 1793, n85). Le célèbre oukase de Catherine II sur le serment que devaient prêter les Français résidant en Russie ne suscite que peu le venin du périodique: il accompagne les textes d'un court passage, où le serment est défini comme "tyrannique », " monument impérissable de la barbarie et du délire des rois », qui « répugne autant à la liberté qu'à la raison » (le 30 mars 1793, $\mathrm{n}^{\circ}$ $89)^{20}$. Et un mois plus tard le journal informe honnêtement ses lecteurs que seuls cinquante Français « refusèrent courageusement au serment prescrit " (le $1^{\mathrm{er}}$ mai 1793 , $\left.\mathrm{n}^{\circ}{ }^{121}\right)^{21}$. Sur ce sujet le journal n'exagère pas l'influence des nouvelles idées libérales sur leurs compatriotes à l'étranger qui préféraient rester dans les contrées du despotisme, mais au calme et en gardant leurs grades, leurs titres et surtout leurs têtes...

A propos de l'arrivée du comte d'Artois à Saint-Pétersbourg, le journal signale que « l'aversion de l'impératrice pour tous les Français, excepté pour ceux qui se sont rendus indignes de porter ce nom, augmente de jour en jour » et que la surveillance sévère des Français en Russie « ressemble à l'inquisition autrichienne » (le 19 février $1793, \mathrm{n}^{\circ} 50$ ). On rencontre ici les concepts clés du «despotisme» et de la "barbarie». Le $M U$ ne les attribue pas à l'Angleterre, malgré l'hostilité entre les deux pays, tandis que la Russie, l'Allemagne et l'Autriche sont régulièrement caractérisées par des épithètes qui soulignent leur ignominie et leur arriération («inquisition autrichienne», « les tyrans de

Constitution danoise du 5 juin 1849. Publication du Ministère Royal des Affaires Etrangères du Danemark, Danemark, 1999, p. 2, 6.

${ }^{19}$ L'aide financière de Catherine II aux émigrés français bénéficie d'une attention vigilante de $M U$. A. Mitrofanov, J. Ollivier-Chakhnovskaïa, art. cit., p. 99.

${ }^{20}$ Le 23 mars 1793, au moment où les Français réfractaires sont expulsés de Russie, la France décrète l'expulsion de tous les prêtres réfractaires au serment civil, ce qui provoque l'arrivée d'environ trois mille prêtres français pour la seule Angleterre, et trois milles quatre cents dans les Iles Anglo-Normandes, sans compter d'autres pays. Simon Burrows, French Exile Journalism and European Politics (1792-1814), Londres, 2001, p. 12. Cet exode n'est pas considéré en France comme une violation de la conscience ou, si l'on emploie les termes du $M U$, un «monument impérissable de la barbarie ». Cette approche démontre encore la partialité du périodique dans la description des événements intérieurs de la « nouvelle » France.

${ }^{21}$ Les données du $M U$ sont très précises : selon de récentes études, soixante-dix Français refusent de prêter le serment et sont expulsés de Russie en 1793, contre 2430 signataires qui acceptent de reconnaître l'essence « usurpatrice » du nouveau gouvernement, "la mort du Roi Très-Chrétien Louis XVI comme un acte de scélératesse abominable et de trahison infâme », de se soumettre aux lois de l'Empire russe et de voir leurs noms publiés dans les journaux, ce qui rendait leur retour en France impossible. Julie OllivierChakhnovskaïa, «Les Français expulsés de l'Empire russe par l'oukase de Catherine II du 8 février 1793 ", Cahiers du Monde russe, 46/3, juillet-septembre 2005, p. 530. 
Darmstadt », « les despotes de Berlin », etc. $)^{22}$. Cette tendance perdurera jusqu'en 1799, où le journal traitera les armées de la coalition d'attroupement d'assassins et de fanatiques $^{23}$. A la même période, la Porte Ottomane, dont la civilisation est éloignée aussi bien de la France que de la Russie ou de l'Autriche, n'est jamais traitée comme un pays « barbare » ou « despotique ». C'est une preuve qu'en 1793 le $M U$ est devenu un organe de propagande face aux ennemis européens de la France. Comme la Porte Ottomane ne présente pas de danger militaire, toutes les divergences sont mises de côté.

\section{La vie sociale et culturelle}

Cependant, le retard de la Russie et le mépris pour ce pays despotique ne sont jamais mis en relation avec le fait religieux: aucune allusion à la "religion grecque », à l'ignorance du clergé ou aux actes "préjudiciables" dictés par la foi ou l'Eglise orthodoxe $^{24}$. Cela contraste, par exemple, avec les moqueries qui parsèment les pages du journal au sujet de la bigoterie des Espagnols ou des Italiens, que l'Eglise instrumentaliserait au profit de la « croisade » antifrançaise. « La raison publique avançait lentement : la voilà qui recule de plusieurs siècles en un jour », écrit le $M U$ le 7 mars 1793 ( $\left.n^{\circ} 66\right)$, en évoquant les « instructions » de l'Angleterre qui a pris part à " cette révolution fanatique » en Espagne ${ }^{25}$. L'Italie est présentée comme un des piliers de l'obscurantisme : « Un fait incontestable, c'est que J.J. Rousseau n'est déjà plus un hérétique dans l'arrière pensée des gouvernements, à Rome même » (le 26 mars 1793, n85).

Les nouvelles provenant des Etats-Unis sont toujours chères aux citoyens français, voilà pourquoi les informations sur ce pays, quoique rares en raison de l'éloignement des deux pays, sont toujours fournies. Vers 1793, dans la rubrique "Etats-Unis d'Amérique », le $M U$ met au point une nouvelle astuce rédactionnelle, afin de populariser les succès de la République Française : ce sont les comptes rendus des toasts portés aux réussites françaises et aux valeurs libérales partagées par les deux pays au cours des fêtes et des réceptions américaines. Le périodique énumère infatigablement et avec un évident plaisir ces toasts fleuris et redondants : par exemple, «puissent la raison, la liberté, l'égalité régner seules sur la terre"; "lois douces et administration vertueuse à tout peuple libre »; «les révolutions de France et d'Amérique. - Puisse l'énergie des patriotes continuer à confondre les prédictions des aristocrates », etc. (le 11 mai $1793, \mathrm{n}^{\circ}{ }_{131}$ ). Les fêtes d'aucun autre pays ne méritent de telles descriptions détaillées, nul toast porté dans une monarchie européenne n'est cité.

Les événements culturels, littéraires, scientifiques en Russie intéressaient toujours très peu le périodique : en 1793, on ne trouve aucune nouvelle de ce type à l'exception peutêtre de l'annonce de la parution du Voyage philosophique, politique et littéraire, fait en Russie, par P.-N. Chantereau (le $1^{\text {er }}$ août $1793, \mathrm{n}^{\circ} 213$ ). L'œuvre est très critique à l'égard de

\footnotetext{
${ }^{22}$ La presse russe, au contraire, à partir de 1792, consacre la plupart de ses pages aux déclarations contrerévolutionnaires du roi de Prusse, des frères de Louis XVI et autres ennemis de la Révolution ; la situation intérieure en France est passée sous un silence presque total. М. Штранге, Русское общество $u$ Французская революция [M. Chtrangué, La société russe et la Révolution Française], Moscou, 1956, p. 117.

${ }^{23}$ К. Мильчин, « Образ России на страницах газеты 'Monituer universel' в 1799 г. », Россия и Франция XVIII-XX вв. [K. Miltchine, «L'image de la Russie dans les pages du Moniteur Universel en 1799 », La Russie et la France XVIII ${ }^{e}-X X^{e}$ siècles], Moscou, 2005, n 6, p. 59. Je remercie Andreï Mitrofanov de m'avoir indiqué cet article.

${ }^{24}$ Dans leurs Mémoires sur la Russie, les Français par contre le soulignent sans fatigue.

${ }^{25}$ Cependant, le périodique n'ajoute aucun commentaire à l'expulsion des Français de Castille (le 22 mars $\left.1793, n^{\circ} 81\right)$.
} 
la Russie et, par conséquent, assez populaire pendant la dictature jacobine ${ }^{26}$, ce qui explique les raisons pour lesquelles le $M U$ insère cette information. Il analyse le contenu du livre, afin de démontrer les défauts du régime monarchique aboli en France : «Ce voyage doit intéresser tous les lecteurs par [...] cet amour vrai et sage de la liberté qui inspire de l'horreur pour le délire de la licence et en peint les abus monstrueux ; par ce zèle, cette fierté, cette franchise avec laquelle l'auteur défend ou fait valoir les droits de l'humanité trop souvent opprimée sous un gouvernement despotique, etc. etc. ». On peut citer un autre livre, dont le contenu et les auteurs sont liés à la Russie : La politique de tous les cabinets de l'Europe, par J.-L. Favier, qui est largement complété par les commentaires de L.-Ph. Ségur, sur la Russie ${ }^{27}$. Cette fois encore, le $M U$ pourfend avec férocité surtout les deux derniers rois français, en critiquant le «secret du Roi », le système qui cependant avait été propre à tous les gouvernements et qui avait servi comme « la résistance aux usurpateurs, à l'influence despotique de la maison d'Autriche et de la Russie, par le moyen d'une alliance avec la Prusse, la Hollande, la Suède, le Danemarck et toutes les puissances secondaires, membres du corps Germanique » (le 17 août ${ }_{1793}, \mathrm{n}^{\circ} 229$ ).

Les nouvelles françaises en 1793 ne sont pas cependant moins «barbares ». Un exemple : les annonces régulièrement parues dans le $M U$ concernant les peines de mort infligées aux « citoyens ", avec leurs noms et les brèves descriptions de leurs « crimes », le plus souvent politiques (propos contre-révolutionnaires, etc.). Ces informations sont souvent insérées juste avant les rubriques «Spectacles », "Livres nouveaux », "Arts ». Tel est le cas du général Adam-Philippe Custine, grand-père du célèbre Astolphe, dont l'exécution est annoncée juste avant la rubrique « Spectacles », dans laquelle l'Académie de Musique invite à assister à l'opéra l'Offrande à la Liberté (le 29 août 1793, n²41).

\section{Conclusion}

Les nouvelles concernant la Russie n'occupent pas beaucoup de place dans les pages du Moniteur universel : ce pays y est présenté comme un état européen parmi d'autres, son rôle dans la balance politique n'est pas exagéré. La vie sociale, scientifique et culturelle de ce vaste Empire est presque ignorée des rédacteurs qui ne s'intéressent qu'à ses intentions vis-à-vis de la France, de ces citoyens, à sa politique internationale et à son état militaire. L’image de la Russie est étroitement liée aux «affaires polonaises » et à ses démarches contre la France révolutionnaire. On ne souligne particulièrement ni l'arriération ni les tares de la civilisation russe. Les critiques à l'encontre de la Russie se focalisent sur son "despotisme » et pas sur sa " barbarie » souvent soulignée dans les écrits des Lumières ; l'image de Catherine II et de son régime dans le $M U$ est loin aussi de celle de la « monarchie éclairée » que nous donne Voltaire. Ces « attaques » ne sont ni plus acerbes ni plus féroces que celles à l'encontre des autres monarchies de l'Europe menaçant la

\footnotetext{
${ }^{26}$ Sur l'œuvre de Chantereau, voir : Michel Cadot, «Le voyage en Russie du citoyen Chantereau ", L'ours et le coq. Essais en l'honneur de M. Cadot réunis par F.-D. Liechtenhan, Paris, 2000, p. 55-66 ; А. Митрофанов, «Образ России в революционной публицистике и периодической печати Франции периода якобинской диктатуры», Россия и Франция, XVIII-XX вв. [A. Mitrofanov, «L'image de Russie dans les écrits publicistes à l'époque de la Révolution et dans la presse périodique pendant la dictature jacobine ", $L a$ Russie et la France XVIII-XX $X^{e}$ siècles], Moscou, 2009, nº 9, p. 81-97.

${ }^{27}$ Sur les opinions de Favier sur la Russie même et les relations franco-russes, voir : F.-D. Liechtenhan, « Le «doux commerce», facteur de rapprochement et de divergence», L'image de l'Etranger, sous la dir. d'Alexandre Stroev, Paris, IES, 2010, p. 35-37.
} 
France révolutionnaire. L’Autriche, la Prusse et la Russie sont rangées parmi les pays « despotiques », tandis qu'un autre ennemi, l'Angleterre, n'en fait pas partie. Le $M U$ n'est ni plus ni moins partial dans le traitement des nouvelles russes que pour les autres ennemis de la République ou que la presse russe à l'égard des affaires françaises après juillet 1789. En général, l'image de la Russie dans ce périodique ressort alternativement soit neutre, soit négative.

\section{Annexe}

Le nombre de titres des pays et des régions mentionnés dans la rubrique « Politique » (Moniteur Universel) en 1790, 1793 et 1796.

I - pour la période janvier-juillet

II - pour la période juillet-décembre

Exemple : 1790 (I) - aux mois de janvier-juillet de l'année 1790

\begin{tabular}{|l|c|c|c|c|c|c|}
\hline & $\begin{array}{c}1790 \\
\text { I }\end{array}$ & $\begin{array}{c}1790 \\
\text { II }\end{array}$ & $\begin{array}{c}1793 \\
\text { I }\end{array}$ & $\begin{array}{c}1793 \\
\text { II }\end{array}$ & $\begin{array}{c}1796 \\
\text { I }\end{array}$ & $\begin{array}{c}1796 \\
\text { II }\end{array}$ \\
\hline Afrique & - & - & 1 & 2 & - & - \\
\hline Allemagne & 88 & 103 & 52 & 46 & 73 & 73 \\
\hline Amérique & - & 3 & - & - & 2 & - \\
\hline $\begin{array}{l}\text { Amérique } \\
\text { septentrionale }\end{array}$ & 4 & 2 & - & - & - & - \\
\hline Angleterre & 88 & 83 & 50 & 26 & 46 & 41 \\
\hline Autriche & - & - & - & - & - & 1 \\
\hline Barbarie & 1 & 1 & - & - & - & 1 \\
\hline Bohême & - & - & - & - & - & 1 \\
\hline $\begin{array}{l}\text { Colonies } \\
\text { françaises }\end{array}$ & 3 & 49 & 1 & 1 & 1 & - \\
\hline $\begin{array}{l}\text { Comtat } \\
\text { vénaissin }\end{array}$ & - & 3 & - & - & - & - \\
\hline Corse & - & 2 & - & - & - & - \\
\hline Danemark & 29 & 29 & 17 & 29 & 12 & 6 \\
\hline Ecosse & - & - & 1 & 1 & - & - \\
\hline Espagne & 30 & 34 & 18 & 6 & 21 & 15 \\
\hline $\begin{array}{l}\text { Etats-Unis } \\
\text { d'Amérique }\end{array}$ & 14 & 8 & 5 & 5 & 13 & 7 \\
\hline Genève & 8 & 5 & 3 & - & - & - \\
\hline Hollande & 10 & 15 & 20 & 9 & - & - \\
\hline Hongrie & - & - & - & - & 1 & 3 \\
\hline Iles Antilles & - & - & - & - & 1 & - \\
\hline Ile de Malte & - & - & 1 & - & - & - \\
\hline Iles du Vent & - & - & 2 & - & - & - \\
\hline $\begin{array}{l}\text { Indes } \\
\text { Occidentales }\end{array}$ & 1 & - & - & - & - & - \\
\hline $\begin{array}{l}\text { Indes } \\
\text { Orientales }\end{array}$ & - & 3 & - & - & - & - \\
\hline Italie & 53 & 30 & 23 & 30 & 54 & 75 \\
\hline
\end{tabular}




\begin{tabular}{|l|c|c|c|c|c|c|}
\hline Irlande & 3 & 1 & 3 & 1 & - & - \\
\hline Liège & 39 & 18 & - & - & - & - \\
\hline Pays-Bas & 96 & 49 & 49 & 30 & - & - \\
\hline Pays de Nice & - & - & 3 & - & - & - \\
\hline Perse & - & - & - & - & 1 & - \\
\hline Piémont & - & - & - & 1 & - & - \\
\hline Pologne & 59 & 52 & 57 & 49 & 15 & 5 \\
\hline Portugal & 23 & 7 & 1 & 1 & 2 & 7 \\
\hline Prusse & 43 & 45 & 14 & 14 & 7 & 9 \\
\hline $\begin{array}{l}\text { République } \\
\text { Batave }\end{array}$ & - & - & - & - & 22 & 25 \\
\hline $\begin{array}{l}\text { République de } \\
\text { Genève }\end{array}$ & - & - & 3 & - & - & - \\
\hline $\begin{array}{l}\text { République } \\
\text { des Provinces }\end{array}$ & - & - & - & - & 4 & - \\
Unies & & & & & & \\
\hline Russie & 24 & 28 & 10 & 17 & 1 & 4 \\
\hline Sardaigne & - & - & 1 & - & - & - \\
\hline Savoie & 4 & 2 & - & - & - & - \\
\hline Serbie & - & - & 1 & - & - & - \\
\hline Suède & 41 & 41 & 45 & 28 & 13 & 12 \\
\hline Suisse & 4 & 8 & 9 & 9 & 7 & 21 \\
\hline Turquie & 53 & 16 & 10 & 12 & 19 & 10 \\
\hline
\end{tabular}

